\title{
Dose Distributions in Simulated Electron Radiotherapy with Intraoral Cones Using Treatment Planning System
}

\author{
Tomohiro Shimozato ${ }^{1}$, Kuniyasu Okudaira ${ }^{2}$ \\ ${ }^{1}$ Department of Radiological Technology, Gifu University of Medical Science, Gifu, Japan \\ ${ }^{2}$ Department of Radiotherapy, Nagoya University Hospital, Aichi, Japan \\ Email: shimo-p@umin.ac.jp
}

How to cite this paper: Shimozato, T. and Okudaira, K. (2017) Dose Distributions in Simulated Electron Radiotherapy with Intraoral Cones Using Treatment Planning System. International Journal of Medical Physics, Clinical Engineering and Radiation Oncology, 6, 280-289.

https://doi.org/10.4236/ijmpcero.2017.63025

Received: July 3, 2017

Accepted: August 4, 2017

Published: August 7, 2017

Copyright $\odot 2017$ by authors and Scientific Research Publishing Inc. This work is licensed under the Creative Commons Attribution International License (CC BY 4.0).

http://creativecommons.org/licenses/by/4.0/

c) (i) Open Access

\begin{abstract}
Aim: This study aims to evaluate the difference between depth data from an intraoral cone and a conventional irradiation tube calculated using a treatment planning system (TPS), and that measured using an intraoral cone for electron radiotherapy. Background: A TPS is only compatible with conventional irradiation tubes. However, such systems are not suitable for determining dose distributions when a special cone is employed. Materials and Methods: Dose distributions were calculated using the beam data for mounted intraoral cones using a TPS. Then, the dose distribution by field size was calculated for a low-melting-point lead alloy using the beam data for a mounted conventional tube. The calculated data were evaluated against the measured intraoral-cone depth data based on the dose and depth differences. Results: The calculated data for the intraoral cone case did not match the measured data. However, the depth data obtained considering the field size determined for the lead alloy using the conventional tube were close to the measured values for the intraoral cone case. The difference in the depth at which the absorbed dose was $50 \%$ of the maximum value of the percentage depth dose was less than $\pm 4 \mathrm{~mm}$ for the generalized Gaussian pencil beam convolution algorithm and less than $\pm 1 \mathrm{~mm}$ for the electron Monte Carlo algorithm. Conclusion: It was found that the measured and calculated dose distributions were in agreement, especially when then electron Monte Carlo algorithm was used. Thus, the TPS can be employed to determine dose distributions for intraoral cone applications.
\end{abstract}

\section{Keywords}

Treatment Planning System, Electron Radiotherapy, Intraoral Cone, Depth Dose, Algorithm 


\section{Introduction}

In external electron radiotherapy, conventional irradiation tubes (applicators) and metallic cones (used in intracavitary [1] and intraoperative [2] irradiation) are employed according to the application requirements, via attachment to the medical linear accelerator (linac) outlet. As regards treatment using an intraoral cone, the irradiation dose is often calculated from tabulated data such as the measured depth data dose and cone factor [3]; thus, the monitor unit (MU) value calculated using the treatment planning system (TPS) is rarely used in clinical scenarios, although the authors have experience with its application.

It is necessary to register the beam data in order to calculate the dose distribution using a TPS. However, a TPS is only compatible with applicators added to the linac, and cannot be used to determine the dose distribution when a special cone, such as an intraoral cone, is employed [4]. Further, the intended dose distribution described to the patient is often different to the actual supplied dose distribution when intraoral cones are used. In fact, Slyk and Litoborski [3] have reported that the dose distribution calculated using the generalized Gaussian pencil beam (GGPB) electron algorithm installed in a commercial Eclipse TPS (Varian Medical Systems, Palo Alto, CA, USA) does not correspond to the measured data when an intraoperative metallic cone is used. Recently, a commercial implementation based on the macro Monte Carlo (MC) method [5] has been developed and has been made available as the electron MC (eMC) dose calculation algorithm in Eclipse. Previous studies have evaluated eMC accuracy with regard to dose distribution prediction for high-energy electron beams [6] [7]. However, although the eMC implementation yields an extremely large improvement when compared with the commonly used pencil beam convolution algorithm, there are some limitations for electron beam energies $\leq 6 \mathrm{MeV}$ [4] [6] [7].

The goal of this study is to evaluate the differences between the depth doses calculated from the beam data obtained using an intraoral cone, along with those calculated from the radiation field determined by a low-melting-point lead alloy (LMA) using the TPS applicator beam data, through comparison with the measured depth doses for an intraoral cone, considering electron beams with energies higher than $6 \mathrm{MeV}$. As a result, we compare measured values and calculated value and evaluate those data. We also consider the usefulness of calculation results using eMC for dose calculation algorithm.

\section{Materials and Methods}

\subsection{Beam Data Measurement}

Electron beams of 6, 9, 12, and $16 \mathrm{MeV}$ emitted from a Clinac 21iX linac (Varian Medical Systems, Palo Alto, CA, USA) were considered, using an applicator (A06; Varian Medical Systems, Palo Alto, CA, USA) employed for conventional electron irradiation (Figure 1(a)) or an intraoral cone (oblique or straight; Engineering System Co., LTD., Japan; Figure 1(b)). The percentage depth 


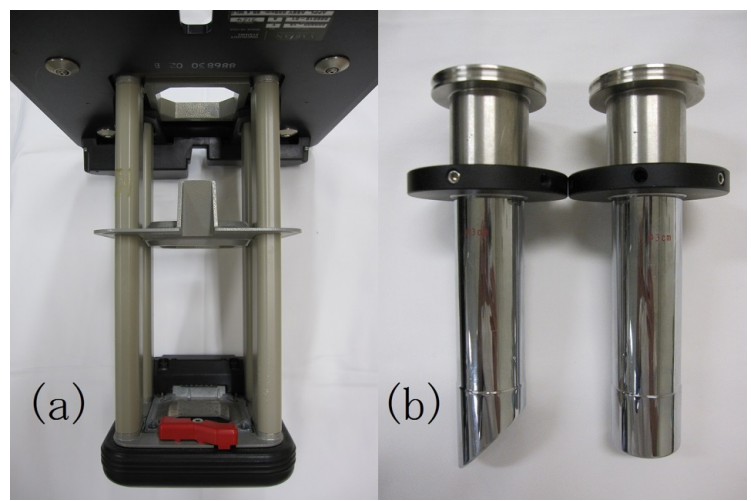

Figure 1. (a) Conventional irradiation applicator and (b) intraoral cones (oblique and straight), which were mounted on the medical linac.

irradiation (PDI) was measured using an MP3 three-dimensional water phantom (PTW Freiburg, Germany) controlled by MEPHYSTO $\mathrm{mc}^{2}$ software (PTW Freiburg, Germany), in accordance with the Eclipse Algorithm Reference Guide [4]. To measure the PDI on the beam axis, a parallel plate ionization chamber (TM34045; Advanced Markus chamber, PTW Freiburg, Germany) and a thimble ionization chamber (TM31016; 3D pinpoint chamber, PTW Freiburg, Germany) were used for the applicator and the intraoral cone cases, respectively. The applied voltages were $-300 \mathrm{~V}$ and $-400 \mathrm{~V}$ for the Advanced Markus and 3D pinpoint chambers, respectively. The $3 \mathrm{D}$ pinpoint chamber used to determine the depth dose when an intraoral cone was employed was set to vertical placement, as the stem parts of the chamber made contact with the cone. A UNIDOS electrometer (PTW Freiburg, Germany) was used as the dosimeter. Note that corrections of the polarity effect and ion recombination were not considered in the measured PDI data. The PDI values were converted into percentage depth doses (PDD) according to the American Association of Physicists in Medicine (AAPM) TG51 protocol [8] using the MEPHYSTO $\mathrm{mc}^{2}$ dose analysis software. The dose rate was $600 \mathrm{MU} / \mathrm{min}$.

The field size when the applicator (A06) was employed was $6 \times 6 \mathrm{~cm}^{2}$ at a source-to-surface distance $(\mathrm{SSD})$ of $100 \mathrm{~cm}$, where the X-ray irradiation target was the source. The secondary collimator (jaw) size was determined for each electron energy. The circle field diameter when the intraoral cone was employed was $20(\mathrm{C} 20)$ or $30 \mathrm{~mm} \varphi(\mathrm{C} 30)$ for the $100-\mathrm{cm} \mathrm{SSD}$. The jaw size when the intraoral cone was employed was set to $5 \times 5 \mathrm{~cm}^{2}$, as recommended by the manufacturer [9]. Two different types of intraoral cone were used, i.e., straight and oblique, where the latter emitted a beam at an angle of $45^{\circ}$ with respect to the beam axis, as shown in Figure 2. Specifically, Figure 2(a) and Figure 2(b) show the geometries for the depth dose measurements performed using the straight and oblique cones, respectively. For the latter, data were obtained parallel to the beam axis and orthogonal to the water surface.

\subsection{Beam Data Modeling}

The beam data were registered to the Eclipse TPS version 13.6.30 (Varian Medi- 


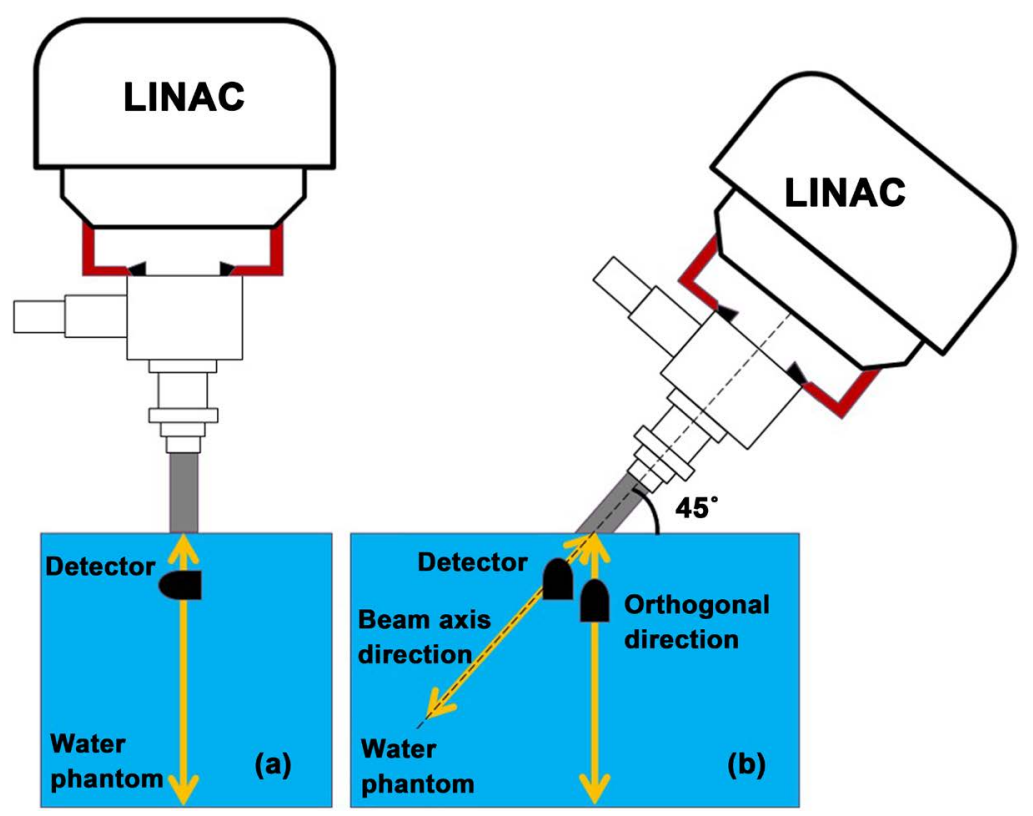

Figure 2. Geometry of water phantom and linac mounted with intraoral cone: (a) straight and (b) oblique cones.

cal Systems Inc., Palo Alto, CA, USA), in accordance with the Beam Configuration Reference Guide [10] and using the beam configuration software supplied with the Eclipse TPS. The data registered with the beam configuration software were the geometrical information, scanning data, and output factor when the applicator was employed. The block transmission data were registered in order to create the radiation field using the LMA and the RT administration software. The transmission factor was registered as 1.0000 .

Then, a new applicator ID was set in order to register the beam data for the intraoral cone case using the RT administration software; this step was implemented because the mechanical specifics of the intraoral cone differ from those of the applicator. The jaw setting when the intraoral cone was implemented was modeled as a $5 \times 5 \mathrm{~cm}^{2}$ field, regardless of the electron energy. Then, the SSD was set to $100 \mathrm{~cm}$. All other information was registered on the RT administration software. The beam configuration registration data were the calculation parameters, dose rate table, electron field-size factors, and the measured depth dose. Maximum and minimum irradiation field (intraoral cone diameters of 20 or 30 $\mathrm{mm} \varphi$ ) sizes were input as the calculation parameters for the 100-cm SSD. The mean incident electron energy was calculated from the equation proposed in IAEA Technical Report No. 381 [11] using the measured PDD. The applicator-skin distance was input as $0.1 \mathrm{~cm}$. The other parameters were equivalent to those for the applicator case. The electron field-size factors were set to 1.0 times the size of a field cell. The output factors were input using the dose rate table applicable to the cell for which the electron field factor was registered as 1.0.

\subsection{Dose Distribution Calculation}

The various dose distributions were calculated using a numeric phantom and the 
external treatment planning software provided with the Eclipse TPS. As noted in the Introduction above, limitations have been reported for electron beam energies of $6 \mathrm{MeV}$ or less when the eMC dose calculation algorithm is implemented. Popple et al. [12] have found differences of up to $5 \%$ between the measured and calculated outputs for $6-\mathrm{MeV}$ electron beams. A similar study has found the same difference (5\%) in the outer regions of the irradiation field for a $6-\mathrm{MeV}$ electron beam and a $15 \times 15 \mathrm{~cm}^{2}$ applicator [13]. Further, Fix et al. [14] have reported that these shortcomings are even more pronounced for a $4-\mathrm{MeV}$ electron beam. Therefore, a $4-\mathrm{MeV}$ electron beam was excluded from investigation in this study, although the medical linac can output such a beam. The dose calculation algorithms were GGPB (version 11.0.31) and eMC (version 13.6.30). The calculation grid sizes were $1.25 \times 1.25 \times 1.25 \mathrm{~mm}^{3}$ and $1.0 \times 1.0 \times 1.0 \mathrm{~mm}^{3}$ for the for GGPB and eMC algorithms, respectively, being the minimum possible values for the respective algorithms. The dose distribution calculations conducted for the applicator and the intraoral cones were performed for gantry angles of $0^{\circ}$ and $45^{\circ}$. The measured depth dose was compared with the depth data calculated from the beam data when an intraoral cone was added, and compared to that calculated from the irradiation determined by the LMA (based on the beam data) when the applicator was mounted. As regards the calculated data for the oblique-type cone, the depth doses on the beam axis and orthogonal to the water surface were evaluated for a $30-\mathrm{mm}$ $\varphi$ irradiation field.

\section{Results and Discussion}

A comparison of the measured and calculated data is shown in Figure 3, for a gantry angle of $0^{\circ}$. As noted in the Materials and Methods section, C20 and C30 indicate the depth doses obtained using the beam data for the intraoral cone case, for irradiation field diameters registered in the TPS as 20 and $30 \mathrm{~mm} \varphi$, respectively. Similarly, A06B20 and A06B30 are the depth doses calculated for irradiation field diameters of 20 and $30 \mathrm{~mm} \varphi$, respectively, as determined by the LMA for the A06 applicator beam data. The error bars for the measured data indicate 3-mm errors in the depth direction (X-axis) and 5\% in the dose direction (Y-axis). The depth doses ( $\mathrm{C} 20$ and $\mathrm{C} 30)$ calculated using the intraoral cone beam data deviate from the error bars in the deeper region approaching the maximum dose depth. Note that the jaw position setting in the Eclipse TPS was determined based on the position recommended for each electron energy by Varian and the irradiated field size on the patient surface. This information was input to the RT administration software in advance. On the other hand, as all inputted beam data registered to the TPS were recognized as those of the applicator, the TPS may not have performed accurate calculations for the beam data obtained using the intraoral cone. Therefore, this inaccuracy is thought to explain the deviation of the calculated data from the measured data.

Note that the depth doses calculated using the irradiation field determined by the LMA based on the applicator beam data were almost within the error bars in the deeper region beyond the maximum dose depth. This TPS dose distribution 

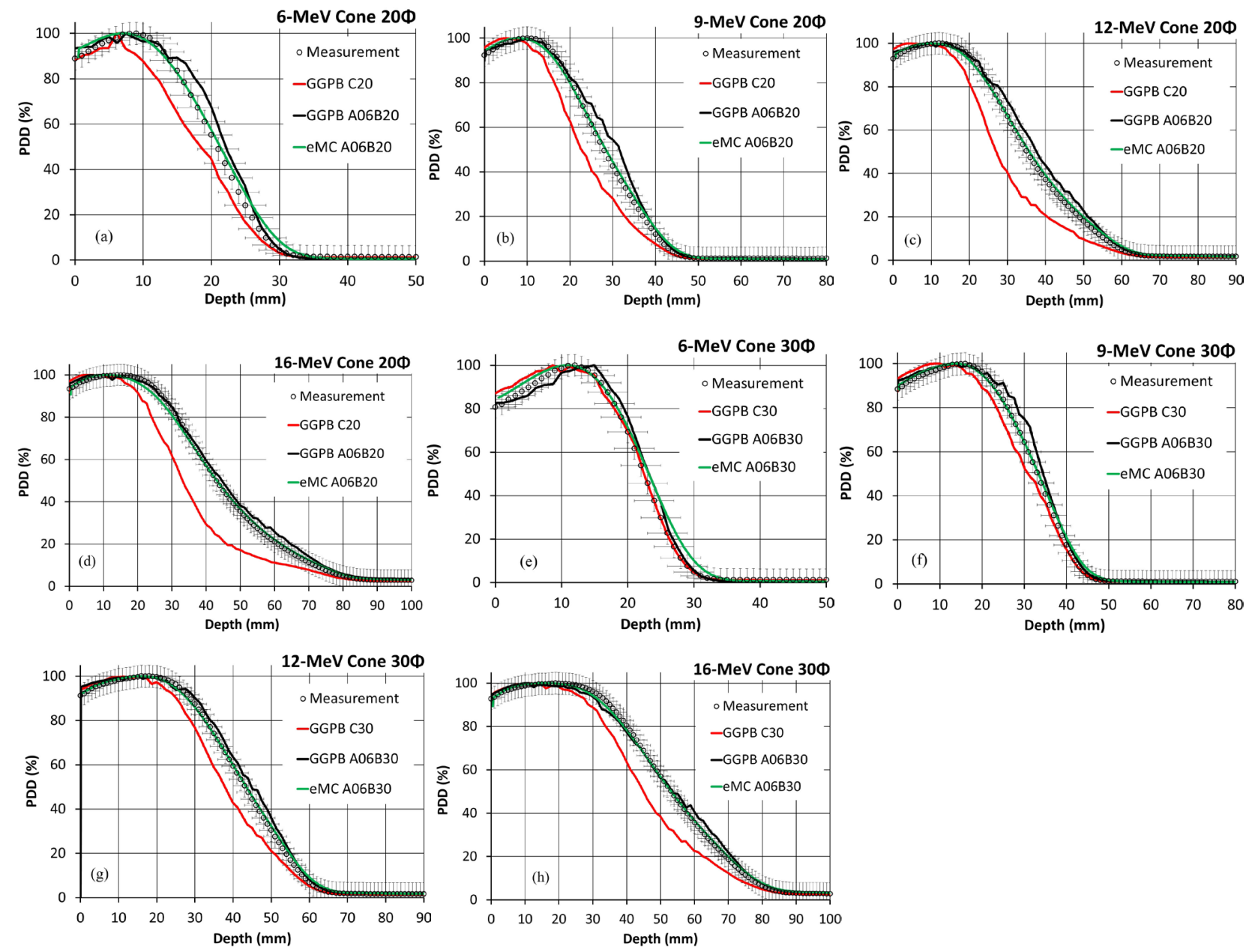

Figure 3. Depth doses using applicator and straight-type intraoral cone for: (a)-(d) 20- and (e)-(h) 30-mm $\varphi$ irradiation field sizes. Depth doses: (a), (e) 6-; (b), (f) 9-; (c), (g) 12-; and (d), (h) 16-MeV. The circle symbols show the measured depth doses. The red and black lines show the depth doses calculated by the GGPB algorithm using the beam data for the intraoral cone and applicator, respectively. The green lines show the depth dose calculated by the eMC algorithm using the applicator beam data. C20 (A06B20) and C30 (A06B30): Data for intraoral cones (A06 applicator) with irradiation field diameters of 20 and $30 \mathrm{~mm} \varphi$, respectively.

calculation was applied to the various field sizes, regardless of the irradiation applicator type.

Table 1 shows the mean difference and standard deviation of each depth dose result, for the beam data for a straight-type intraoral cone and the applicator, as compared to the measurement results. Irradiation cone field sizes of 20 and 30 $\mathrm{mm} \varphi$ and a $100-\mathrm{cm}$ SSD were considered, where the electron beam was orthogonal to the water and along the beam axis, respectively. For the GGPB algorithm and the $20-\mathrm{mm} \varphi$ cone size, the differences in the $\mathrm{R}_{50}$ (the depth in water at which the absorbed dose falls to $50 \%$ of the maximum dose for a certain beam) exceeded $3 \mathrm{~mm}$. As can be seen from the depth dose results shown in Figure 3, the calculated distribution may exhibit a jagged profile in a certain depth region for the GGPB algorithm case. The mean differences and standard deviations for the C20 and C30 cases are extremely large for electron beams with energies more than $9 \mathrm{MeV}$.

The mean differences for the A06B20 and A06B30 cases, as determined using 
Table 1. Mean differences and standard deviations of depth dose results based on beam data for straight-type intraoral cone and applicator, against measurement for irradiation field sizes of 20 and $30 \mathrm{~mm} \varphi$ at the 100-cm SSD. The electron beam was orthogonal to the water and along on the beam axis.

\begin{tabular}{cccccc}
\hline & & \multicolumn{4}{c}{ Difference (\%) } \\
\cline { 3 - 6 } & Algorithm & $6 \mathrm{MeV}$ & $9 \mathrm{MeV}$ & $12 \mathrm{MeV}$ & $16 \mathrm{MeV}$ \\
\hline C20 & GGPB & $9.88 \pm 6.34$ & $9.98 \pm 8.85$ & $11.90 \pm 11.20$ & $12.71 \pm 12.11$ \\
A06B20 & GGPB & $-4.19 \pm 4.92$ & $-3.49 \pm 4.67$ & $-3.26 \pm 3.25$ & $-1.52 \pm 2.07$ \\
A06B20 & eMC & $-1.37 \pm 2.36$ & $-0.40 \pm 1.81$ & $-0.39 \pm 1.51$ & $0.49 \pm 1.68$ \\
C30 & GGPB & $-0.91 \pm 3.11$ & $3.55 \pm 5.51$ & $6.71 \pm 6.86$ & $8.71 \pm 7.93$ \\
A06B30 & GGPB & $-1.37 \pm 4.10$ & $-3.39 \pm 3.67$ & $-2.39 \pm 2.42$ & $-0.01 \pm 2.77$ \\
A06B30 & eMC & $-2.63 \pm 2.69$ & $-0.54 \pm 1.30$ & $-0.16 \pm 1.11$ & $0.35 \pm 1.22$ \\
\hline
\end{tabular}

the GGPB algorithm, were less than $\pm 5 \%$ compared with the measured data. In addition, the mean differences of the A06B20 and A06B30 results, as determined using the eMC algorithm, were less than $\pm 1 \%$ for electron beams with energies higher than $9 \mathrm{MeV}$, as compared with the measured data. Note that, for a $6-\mathrm{MeV}$ electron beam, the accuracy of the calculation data could not be confirmed. However, for a $6-\mathrm{MeV}$ electron beam, the differences between the calculations and measurements were less than $\pm 1.5 \%$.

The depth doses obtained using the oblique-type intraoral cone are shown in Figure 4. In that figure, the measured depth dose, that calculated for the intraoral cone, and that calculated based on the field size determined by the LMA and using the applicator beam data are shown, similar to Figure 3. However, a cone size of $30 \mathrm{~mm} \varphi$ is considered here. As regards the depth dose for the $30-\mathrm{mm} \varphi$ cone size parallel to the beam axis, the intraoral-cone beam data distribution departs from the measured values, except for the $9-\mathrm{MeV}$ case. The results also deviate from the measured distribution when the beam is perpendicular to the water surface. The depth doses by field size as determined by the LMA and using the applicator beam data were limited to within the error bars for all beam energies. The reason for the large differences noted in this figure is that the depth doses were calculated by the GGPB algorithm and the TPS, and it is thought that the scatter components of the lateral calculations were insufficient. Table 2 shows the mean differences and standard deviations for the oblique intraoral cone and applicator data, compared to the measured data when the electron beam was radiated at a $45^{\circ}$ gantry angle. The mean difference for the A06B30 case was less than $\pm 2 \%$ for all algorithms. Further, the standard deviation for the eMC algorithm case was less than that obtained using the GGPB algorithm. The differences in the $\mathrm{R}_{50}$ depths were less than \pm 4 and $\pm 1 \mathrm{~mm}$ for the GGPB algorithm and eMC algorithm, respectively.

\section{Conclusion}

In this study, the differences between the calculated depth doses for two types of intraoral cone and a conventional irradiation applicator were compared to the 

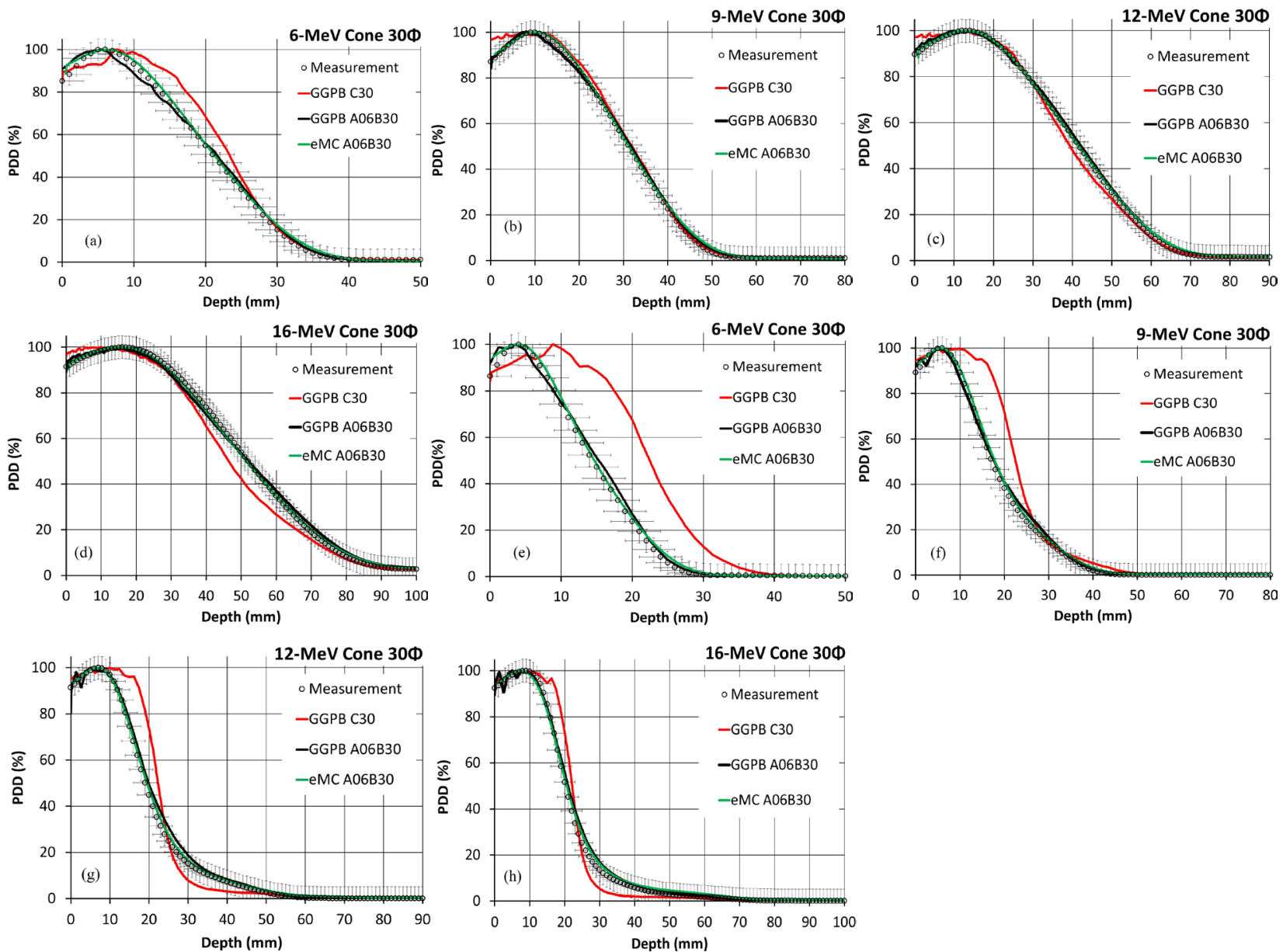

Figure 4. Depth doses using applicator and oblique-type intraoral cone for 30-mm $\varphi$ irradiation cone field size: (a)-(d) Parallel to beam axis; (e)-(h) Orthogonal to water surface. Depth doses for: (a), (e) 6-; (b), (f) 9-; (c), (g) 12-; and (d), (h) 16-MeV. The circle symbols show the measured depth dose. The red and black lines show the depth doses calculated using the GGPB algorithm and the beam data for the intraoral cone and the applicator, respectively. The green lines show the depth dose calculated using the eMC algorithm and the applicator beam data.

Table 2. Mean differences and standard deviations of depth dose results for electron beam along beam axis orthogonal to water surface, using beam data for oblique-type intraoral cone and normal applicator, against measurement for $30-\mathrm{mm} \varphi$ irradiation field size at $100-\mathrm{cm}$ SSD.

\begin{tabular}{ccccccc}
\hline & \multirow{2}{*}{ Algorithm } & \multirow{2}{*}{ Direction } & \multicolumn{4}{c}{ Difference (\%) } \\
\cline { 5 - 7 } & & & $6 \mathrm{MeV}$ & $9 \mathrm{MeV}$ & $12 \mathrm{MeV}$ & $16 \mathrm{MeV}$ \\
\hline C30 & GGPB & Beam axis & $-5.96 \pm 6.79$ & $-2.29 \pm 1.88$ & $-1.14 \pm 3.18$ & $4.64 \pm 5.03$ \\
A06B30 & GGPB & Beam axis & $-0.05 \pm 2.63$ & $-0.21 \pm 1.26$ & $-0.76 \pm 0.99$ & $0.39 \pm 2.04$ \\
A06B30 & eMC & Beam axis & $-1.33 \pm 1.13$ & $-0.74 \pm 0.68$ & $-0.16 \pm 0.65$ & $0.51 \pm 0.85$ \\
C30 & GGPB & Orthogonal & $-21.22 \pm 20.38$ & $-15.55 \pm 14.08$ & $-11.18 \pm 12.04$ & $-6.53 \pm 8.93$ \\
A06B30 & GGPB & Orthogonal & $-1.88 \pm 2.74$ & $-0.96 \pm 2.41$ & $-2.57 \pm 2.76$ & $-1.35 \pm 2.93$ \\
A06B30 & eMC & Orthogonal & $-1.74 \pm 1.10$ & $-1.78 \pm 1.11$ & $-1.04 \pm 1.37$ & $-0.52 \pm 2.34$ \\
\hline
\end{tabular}

measured data for an intraoral cone, for electron beams with energies higher than $6 \mathrm{MeV}$. The depth doses calculated based on the field size determined by 
the LMA and using the applicator beam data approached the measured depth dose for the applicator case. Although it was not necessary to register the depth data obtained using the intraoral cone with the RT administration software, those data were used to confirm the depth dose calculated by the Eclipse TPS. It was found that the dose distribution calculated by the Eclipse TPS reflects the actual distribution for the intraoral cone. Thus, dose distributions planned by the TPS can be employed, especially when the eMC algorithm is used for the calculation. However, the calculated dose distribution was confirmed using Eclipse TPS version 13 only; therefore, it is necessary to confirm the differences between the calculated and measured data for other TPS.

\section{Financial Disclosure}

No funding or affiliation to disclose.

\section{Conflict of Interest Statement}

The authors have no COI to report.

\section{References}

[1] Wexler, M.C., Tobochnik, N. and Spiegler, P. (1982) Characteristics of Intraoral Cone for Electron Beam Therapy with an $18 \mathrm{MeV}$ Linear Accelerator. International Journal of Radiation Oncology Biology Physics, 8, 2001-2004. https://doi.org/10.1016/0360-3016(82)90461-8

[2] Bedder, A.S. and Krishnan, S. (2005) Intraoperative Radiotherapy Using a Mobile Electron LINAC: A Retroperitoneal Sarcoma Case. Journal of Applied Clinical Medical Physics, 6, 95-107. https://doi.org/10.1120/jacmp.v6i3.2109

[3] Slyk, M. and Litoborski, M. (2010) Verification in the Water Phantom of the Irradiation Time Calculation Done by the Algorithm Used in Intraoperative Radiotherapy. Report of Practical Oncology and Radiotherapy, 15, 132-137. https://doi.org/10.1016/j.rpor.2010.08.007

[4] Varian Medical Systems (2011) Eclipse Algorithm Reference Guide. P/N B503486R01B.

[5] Neuenschwander, H. and Born, E.J. (1992) A Macro Monte Carlo Method for Electron Beam Dose Calculations. Physics in Medicine and Biology, 37, 107-125. https://doi.org/10.1088/0031-9155/37/1/007

[6] Ding, G.X., Duggan, D.M., Coffey, C.W., Shokrani, P. and Cygler, J.E. (2006) First Macro Monte Carlo Based Commercial Dose Calculation Module for Electron Beam Treatment Planning-New Issues For Clinical Consideration. Physics in Medicine and Biology, 51, 2781-2799. https://doi.org/10.1088/0031-9155/51/11/007

[7] Pemler, P., Besserer, J., Schneider, U. and Neuenschwander, H. (2006) Evaluation of a Commercial Electron Treatment Planning System Based on Monte Carlo Techniques (eMC). Zeitschrift fur Medizinische Physik, 16, 313-329. https://doi.org/10.1078/0939-3889-00330

[8] Almond, P.R., Biggs, P.J., Coursey, B.M., Hanson, W.F., Huq, M.S., Nath, R. and Rogers, D.W.O. (1999) AAPM's TG-51 Protocol for Clinical Reference Dosimetry of High Energy Photon and Electron Beams. Medical Physics, 26, 1847-1870. https://doi.org/10.1118/1.598691

[9] Engineering System Co. LTD. (2010) Instruction Manual: Electron Beam Irradia- 
tion Guide.

[10] Varian Medical Systems (2011) Beam Configuration Reference Guide. P/N B503483R01A.

[11] International Atomic Energy Agency (IAEA) (1997) The Use of Plane Parallel Ionization Chambers in High Energy Electron and Photon Beams: An International Code of Practice for Dosimetry. Technical Report Series No. 381.

[12] Popple, R.A., Weinberg, R., Antolak, J.A., Ye, S.J., Pareek, P.N., Duan, J., Shen, S. and Brezovich, I.A. (2006) Comprehensive Evaluation of a Commercial Macro Monte Carlo Electron Dose Calculation Implementation Using a Standard Verification Data Set. Medical Physics, 33, 1540-1551. https://doi.org/10.1118/1.2198328

[13] Brualla, L., Palanco-Zamora, R., Wittig, A., Sempau, J. and Sauerwein, W. (2009) Comparison between PENELOPE and Electron Monte Carlo Simulations of Electron Fields Used in the Treatment of Conjunctival Lymphoma. Physics in Medicine and Biology, 54, 5469-5481. https://doi.org/10.1088/0031-9155/54/18/008

[14] Fix, M.K., Cygler, J.E., Frel, D., Volken, W., Neuenschwander, H., Born, E.J. and Manser, P. (2013) Generalized eMC Implementation for Monte Carlo Dose Calculation of Electron Beams from Different Machine Types. Physics in Medicine and Biology, 58, 2841-2859. https://doi.org/10.1088/0031-9155/58/9/2841

Submit or recommend next manuscript to SCIRP and we will provide best service for you:

Accepting pre-submission inquiries through Email, Facebook, LinkedIn, Twitter, etc. A wide selection of journals (inclusive of 9 subjects, more than 200 journals)

Providing 24-hour high-quality service

User-friendly online submission system

Fair and swift peer-review system

Efficient typesetting and proofreading procedure

Display of the result of downloads and visits, as well as the number of cited articles

Maximum dissemination of your research work

Submit your manuscript at: http://papersubmission.scirp.org/

Or contact ijmpcero@scirp.org 